\section{Endocytoscopy assists in the intraoperative diagnosis of carcinoma in a patient with chronic pancreatitis}

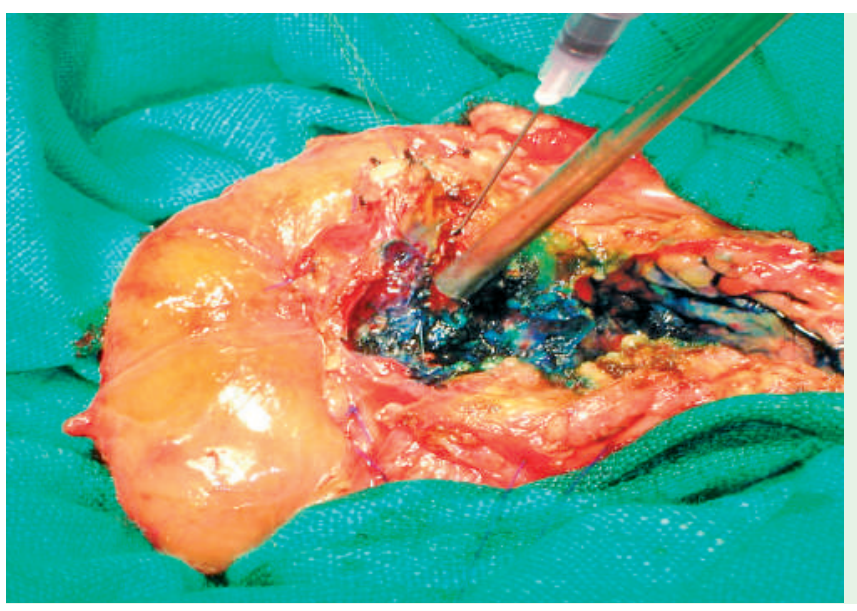

Fig. 1 Intraoperative photograph of the head and body of the pancreas, showing spraying of the opened pancreatic duct with methylene blue dye.

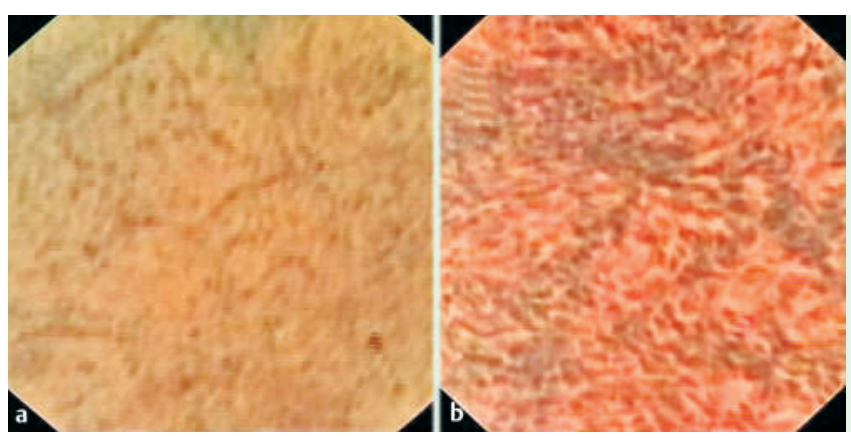

Fig. 2 Intraoperative cytoscopic images of the pancreatic duct in the pancreatic body/ tail region (a) and in the pancreatic head (b).

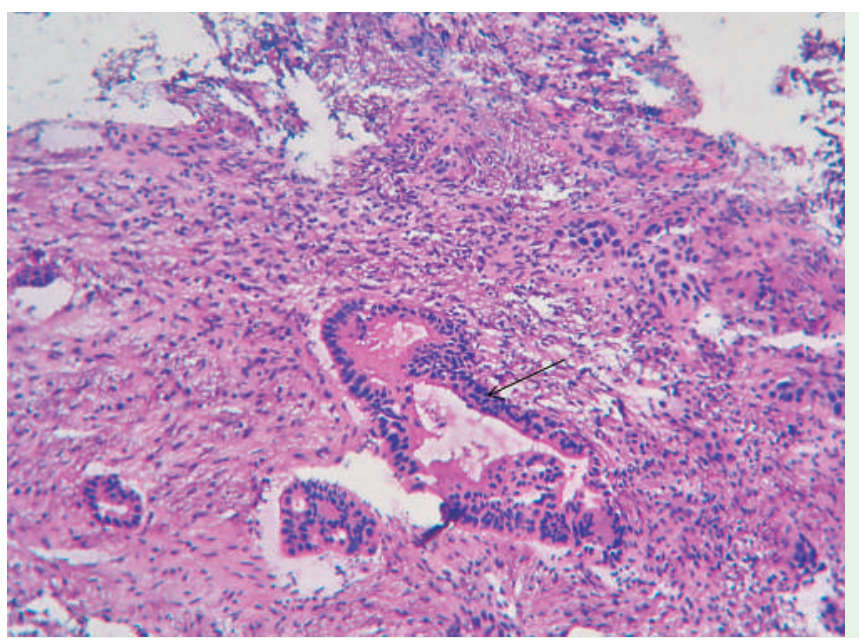

The endocytoscopy system has been used successfully for direct histological imaging of the gastrointestinal mucosa during endoscopy [1 -3]. However, there are no published reports on the intraoperative use of the endocytoscope. This is the first report describing the intraoperatiave use of the endocytoscope for the detection of carcinoma in a patient with chronic pancreatitis.

A 44-year-old man with longstanding chronic pancreatitis presented with recurrent episodes of abdominal pain and significant weight loss. His CA19-9 level was raised at $392 \mathrm{U} / \mathrm{mL}$ (normal range 0-25 U/mL). Computed tomography showed changes typical of chronic pancreatitis, but no evidence of tumor. Elective pancreaticojejunostomy was planned because there were multiple strictures in the pancreatic duct. Intraoperatively, the head of pancreas was bulky but there was no palpable mass. The pancreatic duct was opened and showed a long stricture with ulcerations in the head region. It was decided that endocytoscopic evaluation might be useful in order to assess the type and extent of the lesion. The opened pancreatic duct was sprayed with sterilized methylene blue dye ( Fig. 1). The body region of the pancreatic duct was examined first in order to familiarize ourselves with the normal cytoanatomy of the duct, and then we looked at the suspicious area in the head region, which showed clusters of tumor cells with hyperchromatic nuclei and a high nuclear-cytoplasmic ratio ( $\bullet$ Fig. 2). This pattern was found only in the head of the pancreas. A biopsy of this tissue was sent for frozen section examination and subsequently for conventional histopathological examination, which showed malignancy, confirming the endocytoscopy findings ( $\bullet$ Fig. 3 ).

In conclusion, endocytoscopy assisted in making a diagnosis of carcinoma on a background of chronic pancreatitis, by visualizing variation in cell size, disorder of polarity, and deformity of the nuclei. This optical biopsy technique could be useful for the immediate diagnosis of mitotic lesions, definition of the extent of these lesions, and assessment of the negativity of resection margins during surgery.

Endoscopy_UCTN_Code_CCL_1AZ_2AB 
G. V. Rao, R. Pradeep,

M. J. Mansard, C. Ramji, R. Banerjee,

D. Nageshwar Reddy

Department of Gastroenterology, Asian

Institute of Gastroenterology, Hyderabad,

Andhra Pradesh, India

\section{References}

1 Inoue H, Kudo SE, Shiokawa A. Novel endoscopic imaging techniques toward in vivo observation of living cancer cells in the gastrointestinal tract. Dig Dis 2004; 22: 334 337

2 Inoue $H$, Sasajima K, Kaga $M$ et al. Endoscopic in vivo evaluation of tissue atypia in the esophagus using a newly designed integrated endocytoscope: a pilot trial. Endoscopy 2006; 38: $891-895$

3 Sasajima K, Kudo S, Inoue $H$ et al. Real-time in vivo virtual histology of colorectal lesions when using the endocytoscopy system. Gastrointest Endosc 2006; 63: 1010- 1017
Bibliography

DOI 10.1055/s-2007-966798

Endoscopy 2007; 39: E317-E318

(c) Georg Thieme Verlag KG Stuttgart · New York . ISSN 0013-726X

\section{Corresponding author}

\section{Nageshwar Reddy, MD}

Asian Institute of Gastroenterology

6-3-661 Somajiguda

Hyderabad 500082

Andhra Pradesh

India

Fax: +91-40-2332-4255

aigindia@yahoo.co.in 